\title{
Human-Computer Interaction, Ethics, and Biomedical Informatics
}

\author{
Harry Hochheiser', Rupa S. Valdez² \\ 1 Department of Biomedical Informatics, University of Pittsburgh School of Medicine, Pittsburgh, \\ Pennsylvania USA \\ 2 Public Health Sciences \& Engineering Systems and Environment, University of Virginia, \\ Charlottesville, Virginia USA
}

\begin{abstract}
Summary
Objectives: To provide an overview of recent work at the intersection of Biomedical Informatics, Human-Computer Interaction, and Ethics.

Methods: Search terms for Human-Computer Interaction, Biomedical Informatics, and Ethics were used to identify relevant papers published between 2017 and 2019. Relevant papers were identified through multiple methods, including database searches, manual reviews of citations, recent publications, and special collections, as well as through peer recommendations. Identified articles were reviewed and organized into broad themes.

Results: We identified relevant papers at the intersection of Biomedical Informatics, Human-Computer Interactions, and Ethics in over a dozen journals. The content of these papers was organized into three broad themes: ethical issues associated with systems in use, systems design, and responsible conduct of research.

Conclusions: The results of this overview demonstrate an active interest in exploring the ethical implications of Human-Computer Interaction concerns in Biomedical Informatics. Papers emphasizing ethical concerns associated with patient-facing tools, mobile devices, social media, privacy, inclusivity, and e-consent reflect the growing prominence of these topics in biomedical informatics research. New questions in these areas will likely continue to arise with the growth of precision medicine and citizen science.
\end{abstract}

\section{Keywords}

User-computer interface, ethics, medical informatics

Yearb Med Inform 2020:93-8

http://dx.doi.org/10.1055/s-0040-1701990

\section{Introduction}

Ethics in Human-Computer Interaction (HCI) covers a wide range of topics including: human welfare, ownership and property, privacy, freedom from bias, universal usability, trust, autonomy, informed consent, and accountability, among others [1]. Like HCI, biomedical informatics research and practice is deeply concerned with the ethical implications of technology design and use. The code of professional and ethical conduct of the American Medical Informatics Associaion (AMIA), revised in 2018, highlights topics such as informed use and control of data by patients, security and privacy, respect for human participants in research, and awareness of social or public health implications as aspects of ethical behavior [2]. Although the scope of ethics in these fields can be broadly conceptualized as described above, certain key themes emerge as prevalent within the literature during any given time period. To provide an overview of topics at the intersection of biomedical informatics, HCI and ethics, we explored literature published in years 2017-2019.

\section{Methods}

We surveyed recent literature to provide an overview of key themes at the intersection of biomedical informatics, HCI, and ethics. We started with an examination of recent publications, which were used to identify search terms for both HCI and ethics. The
HCI search terms included "human-computer interaction" and variants along with additional terms discussing interfaces, displays, user-centered design, and virtual systems. Ethics search terms included topics such as bias, bioethics, security, confidentiality, privacy, disabilities, consent, legislation, autonomy, personhood, privacy, racism, vulnerable populations, underserved, and related variants. The HCI search terms were combined with the ethics terms in conjunctive searches, with the addition of a temporal term restricting results to papers published between January 1, 2017 and December 31, 2019 (to include electronic publications ahead of print). Additional articles were identified through manual review of recent publications in medical informatics journals, recommendations from colleagues, and review of curated collections including James Cimino's 2019 Year-in-Review presentation at AMIA 2019 [3], the Journal of Medical Internet Research's e-collection "Ethics, Privacy, and Legal Issues" for the years 2017 to 2019 [4], the special issue of the Journal of American Medical Informatics Association (JAMIA) on Health Informatics and Health Equity published in August-September 2019 [5], and through further manual searches and review of citations. Between October and December 2019, we engaged in an ongoing dialogue about this literature both with each other and with our informatics colleagues. Our reading of the literature combined with these dialogues led to the identification of overarching themes, which we present in the synthesis below. 


\section{Results}

Three broad topics emerged from our reading of and dialogue about the last three years of work at the intersection of biomedical informatics, HCI, and ethics. These themes can be characterized as 1) Systems in Use, 2) System Design, and the overlapping but distinct topic of tools, and 3) Responsible conduct of research. Under our discussion of each of these themes, we highlight the breath of relevant literature, giving particular attention to the range of ethical considerations most prominently discussed in the literature.

\section{1) Systems in Use}

Studies of the implications of systems in use draw on experience with deployed tools to identify ethical issues generally not anticipated prior to system development or deployment. Using both quantitative and qualitative analysis methods, these studies attempt to look back at notable incidents, failures, or simply a body of experiences with a system in situ, in the hope of identifying issues or perspectives that might help avoid future difficulties.

\section{Clinical Informatics}

Potentially adverse impacts of poor usability of electronic health records (EHRs) continue to be a concern, particularly with respect to patient safety. Consistent with earlier efforts [6-9], examinations of patient safety reports continue to find evidence of a strong impact of usability problems on EHR safety [10,11]. A laboratory study of two commonly-used EHRs in four healthcare systems found wide variability in task completion time, required clicks, and error rates (ranging from $0 \%$ to $50 \%$ for various tasks), even though the products had been certified by accreditation authorities [12]. Overly burdensome and often inconsistent EHR documentation practices have also been discussed as a potential source of physician stress and safety risks [13-15]. Whether or not regulatory frameworks for EHRs might be sufficiently mature to address usability and safety concerns was explored by comparing the Office of the
National Coordinator's (ONC) policies for EHRs with analogous policies issued by the Federal Drug Administration (FDA) and Federal Aviation Administration (FAA). FDA and FAA policies were found to be more robust and prescriptive than those of the ONC [16]. Some observers have called for regulatory measures, including national tracking of EHR usability and safety problems, publication of design standards, development of standard usability and safety measurement scenarios [17], increased transparency in researching and sharing EHR usability and safety issues [18], and recommitment to shared responsibility in improving EHR use [19-21].

Usability difficulties in related clinical systems may influence the reliability of some of the data used to understand potential safety hazards associated with EHR use. A systematic review of 48 patient safety event reporting tools identified usability issues such as omission of input validation facilities and hierarchical data layout as frequently found shortcomings [22]. Although the authors do not speculate as to the exact nature and magnitude of the impact of these difficulties, it seems possible that usability problems might lead to inaccurate and incomplete reports, and subsequently to undercounting safety events.

Personal Health Informatics: PHRs, Portals, and Personal Health Information Management

Patient portals bring a different set of challenges, particularly since usability or interaction design shortcomings may effectively disenfranchise patients who find the tools difficult to use or even inaccessible [23]. Encouragement from providers, perceived possibilities of greater access to health information, and improved communication can facilitate portal use, while lack of awareness, lack of training, and privacy/ security concerns present barriers [24], although others have noted that focusing on individual concerns might obscure systemic inequities that discourage use [25]. However, experience with the OpenNotes platform suggests that direct sharing of notes through patient portals might be beneficial. A survey of almost 30,000 Open-
Notes users from three health care systems found that patients found notes helpful for managing their health, with relatively low rates of confusion and substantial benefits among potentially vulnerable populations, including those with lower education levels and non-native English speakers [26].

\section{Social Media and Online Communities}

The majority of articles focused on ethical concerns at the intersection of social media and biomedical informatics published during the review period focus on the ethical conduct of research on and through these platforms [27-33], including consideration for particularly sensitive cases in mental health $[34,35]$. A few articles discuss the ethical issues associated with direct use of social media for health by patients and providers as they relate to HCI [36, 37]. Social media systems often do not clearly indicate the extent to which healthcare providers access and attend to information posted by patients on social media platforms. As a result, patients may post information that they would otherwise not want their provider to see without realizing that a provider may be able to access this information $[31,36]$. Conversely, patients may post information believing that a health-care provider both has access to and will respond to the post. Both cases underscore the need for designs that clearly signal both potential and actual consumption of and responsiveness to posted information by healthcare providers and other individuals. Another key issue relates to information interpretation by patients and providers, who face challenges in interpreting comprehensiveness, accuracy, completeness, and authenticity of information posted on social media platforms $[36,37]$. The presence of both missing information and misinformation generated by both individuals and automated bots emphasizes the need to design systems that use clear communication of potential risks to mitigate adverse consequences [ 38 , 39]. The use of social media for targeted advertising, particularly for sensitive topics such as mental health services, presents additional questions as to which applications of these data are considered to be 
acceptable [40]. A final concern relates to patient understanding of privacy risks as communicated by complex, evolving, and opaque terms and conditions on social media platforms [36, 39]. There is significant opportunity for deeper understanding and intervention from both the HCI and biomedical informatics communities across these areas of concern.

\section{Mobile}

Mobile health apps present several ethical concerns. Prominent among these concerns are privacy and security, particularly when personal health data is involved [41-45]. Examinations of these questions from the perspectives of specific sub-groups, such as men who have sex with men [46] or under-represented ethnic groups [47] emphasize the importance of understanding the needs of specific populations. There is also a need to consider privacy and security as it relates to unintended users. For example, bystanders of an individual using an app intended to capture illicit drug use may inadvertently, without their consent, share elements such as their location, voice, topic of conversation, and other data elements [48].

Clinical applications present similar ethical issues. Although initial data from a small survey suggests that patients might be willing to share data from their devices for use in mental health assessment [49], the use of mobile apps to directly provide psychotherapy and more general mental health support [50] raises concerns about accountability and privacy as well as the need for such systems to explicitly acknowledge the limitations of the source of advice [51]. In an echo of some of the earliest discussions of concerns over the impact of artificial intelligence [52, 53], the emergence of automated chatbot therapy apps has led to calls for ethical standards concerning privacy and the need for disclosure of the automated nature of the tools [54]. The use of mobile devices for communication of other health-related information, such as medication descriptions, have similarly raised concerns about effective communication, usability, and appropriate regulation [55].

\section{2) Systems Design}

Projects in this category differ from discussions of systems in use in that they involve development of novel systems design with the explicit goal of meeting previously unfulfilled ethical considerations. To reach this goal, these projects may be associated with qualitative up-front efforts aimed at understanding relevant needs and preferences and developing designs to account for them.

Perhaps unsurprisingly, attempts to address ethical concerns through novel designs continue to be challenged by the complexities of clinical users and contexts. Preliminary studies of an EHR system that uses pixelation to protect privacy by hiding sensitive information found the design might have improved understanding of privacy concerns. However, difficulties with the inconvenience of revealing the information and the possibility of missing important information raised concerns among potential users [56]. Similarly, a proposed privacy protection design has provided patients with facilities for redacting sensitive content from EHR documents. Although preliminary focus group evaluations found that users had appropriate mental models, several novel requirements were identified, including the need for establishment of trust and clear communication of the handling of redacted data [57].

The importance of considering the perspectives of distinct patient populations is seen in a range of efforts, including preliminary inquiries into potential designs for managing opioid abuse risk in military settings [58], teen smoking prevention tools [59], electronic system decision aids for depression [60], apps for menstrual tracking [61], personal health records for young adults leaving foster care [62], and identification of research areas for informatics support of families experiencing challenges of hospitalization and subsequent care [63]. A proposed clinical trial of the potential use of computer-delivered advice for encouraging physical activity in underserved populations [64] illustrates issues at the intersection of trust, inclusivity, and HCI, as different groups may have different responses to these automated agents. Although social media may play a role, as in a project using analysis of blog posts to understand the sentiments and attitudes of individuals undergoing gender transitions [65], in-depth work with individuals and groups representing these diverse perspectives is likely to be critical to both successful design and responsible use of informatics tools.

\section{3) Research Conduct}

Efforts in this category focus on the development of interactive tools in the support of appropriate conduct of research activities, primarily focusing on outreach and consent to participate in research. As identifying potential participants and helping them become better-informed about the implications of their participation are key components of ethical research conduct, questions of trust and related issues of fairness are not far behind.

\section{Consent, Trust, and Participant Engagement}

Tablet, mobile, or portal-based systems present new opportunities for effectively providing educational materials necessary for informed patient and research study consent. Qualitative studies assessing user needs and preferences have suggested that these tools might effectively address user concerns and potentially increase participation among under-represented groups [66, 67]. However, evaluations have not always found clear wins, with some studies failing to see improvements associated with the use of e-consent [68], and others finding difficulties with complex content [69]. Other efforts have explored interface designs intended to slow users down in the hopes of increasing comprehension [70], and the use of learning theory to design consent content [71]. A review of research on consent processes for research mediated by mobile apps found a range of practices, including some not found in traditional consent, such as assessments of consent understanding and the use of finger-drawn singatures [72]. The broad range of delivery methods, study designs, and assessments used in these studies suggests a need for further work to better understand which approaches might work best in which contexts. 


\section{Recruiting}

Informatics approaches can contribute to the reduction of bias in research studies, as seen in a study that found that patients excluded from a medical device trial due to lack of computer or internet access were among those who might have been most in need of the intervention [73]. Changes to recruiting practices might address some of the imbalances in research participation, as seen in a large trial involving the use of a patient portal to invite participation in a research recruitment registry. Portal invitations led to appropriate representation for women, but differences for Black males, Hispanics, and Asians persisted [74]. A similar approach aimed at urban African-Americans embedded recruiting information in personalized lists of health-related community resources, with encouraging preliminary results, although overall recruitment rates remained low [75]. The PRIDE study, which used in-depth efforts from advisory panels and outreach ambassadors to guide the design of a research platform for a cohort study of sexual and gender minority people [76], provides an example of how a commitment toward understanding and working with underserved or otherwise historically marginalized communities on both the explication of information needs and subsequent design responsive to such needs are critical to successful engagement in research.

Recruitment through social media is also a possibility. A survey of Twitter users and participants on a crowdsourcing platform found that most users might not object to monitoring Twitter content to find study participants, but variations in preferences suggest a need for caution [77]. Similar concerns informed the development of a privacy-by-design framework for using social-media to identify study participants [31].

\section{Conclusions}

Our reading of the last three years of scholarship at the intersection of biomedical informatics, $\mathrm{HCI}$, and ethics demonstrates an active dialogue about the issues. The value placed upon such discussions is exemplified by the robust collection of articles identified through this overview and published in over a dozen journals, including special issues on the topic in both JAMIA and the Journal of Medical Internet Research (JMIR). The three themes identified provide a useful starting point for considering the breadth of work at this intersection, namely systems in use, system design, and research conduct. The publications of the last several years emphasize the need for future scholarship particularly in mobile health and social media, inclusivity, and e-consent. As informatics applications expand into emergent domains, so too will the range of ethical considerations that the field will need to address. For example, new questions related to privacy, welfare, and inclusivity, among others, will result from the rise of precision medicine and citizen science [78]. Similarly, the rapid rise of mobile tracking as a mechanism for public health surveillance and telemedicine in the wake of the COVID-19 pandemic will necessitate additional debates at the intersection of biomedical informatics, HCI, and ethics $[79,80]$. Given this dynamic landscape, there will be an ongoing need not only to engage in ethical discussions but to synthesize and present them in ways that are accessible to the biomedical informatics community.

\section{Acknowledgments}

Charles B. Wessel of the University of Pittsburgh Health Sciences Library System provided assistance with the development of literature search queries.

\section{References}

1. Friedman B, Kahn PH. Human values, Ethics, and Design. In: Jacko J, Sears, A, editors. Hum-Comput Interact Handb. Mahwah, N.J.: Lawrence Erlbaum Associates; 2002. p. 1177-1201.

2. Petersen C, Berner ES, Embi PJ, Fultz Hollis K, Goodman KW, Koppel R, et al. AMIA's code of professional and ethical conduct 2018. J Am Med Inform Assoc 2018 Nov 1;25(11):1579-82.

3. Cimino JJ. Biomedical and Health Informatics Year in Review: Putting the Working Groups to Work [Internet]. 2019 [cited 2019 Nov 20] https:// uab.app.box.com/s/7rk7iicznc29r6h5pwdpumbt$4 \mathrm{f} 5 \mathrm{ba} 8 \mathrm{wb}$

4. JMIR - E-collection "Ethics, Privacy, and Legal Issues" [Internet]. [cited 2019 Nov 21] https:// www.jmir.org.

5. Veinot TC, Ancker JS, Bakken S. Health informatics and health equity: improving our reach and impact. J Am Med Inform Assoc 2019 Aug 1;26(8-9):689-95.

6. Meeks DW, Smith MW, Taylor L, Sittig DF, Scott JM, Singh H. An analysis of electronic health record-related patient safety concerns. J Am Med Inform Assoc 2014 Dec;21(6):1053-9.

7. Magrabi F, Ong M-S, Runciman W, Coiera E. An analysis of computer-related patient safety incidents to inform the development of a classification. J Am Med Inform Assoc 2010 Dec;17(6):663-70.

8. Magrabi F, Baker M, Sinha I, Ong M-S, Harrison S, Kidd MR, et al. Clinical safety of England's national programme for IT: a retrospective analysis of all reported safety events 2005 to 2011 . Int J Med Inform 2015 Mar;84(3):198-206.

9. Wang J, Liang H, Kang H, Gong Y. Understanding Health Information Technology Induced Medication Safety Events by Two Conceptual Frameworks. Appl Clin Inform 2019 Jan;10(1):158-67.

10. Palojoki S, Mäkelä M, Lehtonen L, Saranto K. An analysis of electronic health record-related patient safety incidents. Health Informatics J 2017;23(2):134-45.

11. Ratwani RM, Savage E, Will A, Fong A, Karavite D, Muthu N, et al. Identifying Electronic Health Record Usability And Safety Challenges In Pediatric Settings. Health Aff Proj Hope 2018;37(11):1752-9.

12. Ratwani RM, Savage E, Will A, Arnold R, Khairat S, Miller K, Fairbanks et al. A usability and safety analysis of electronic health records: a multi-center study. J Am Med Inform Assoc 2018 01;25(9):1197-201.

13. Weiner M. Forced Inefficiencies of the Electronic Health Record. J Gen Intern Med 2019 Nov 1;34(11):2299-301.

14. Cohen GR, Friedman CP, Ryan AM, Richardson CR, Adler-Milstein J. Variation in Physicians' Electronic Health Record Documentation and Potential Patient Harm from That Variation. J Gen Intern Med 2019 Nov 1;34(11):2355-67.

15. Flanagan ME, Militello LG, Rattray NA, Cottingham AH, Frankel RM. The Thrill Is Gone: Burdensome Electronic Documentation Takes Its Toll on Physicians' Time and Attention. J Gen Intern Med 2019 Jul;34(7):1096-7.

16. Savage EL, Fairbanks RJ, Ratwani RM. Are informed policies in place to promote safe and usable EHRs? A cross-industry comparison. J Am Med Inform Assoc 2017 Jul 1;24(4):769-75.

17. Ratwani RM, Reider J, Singh H. A Decade of Health Information Technology Usability Challenges and the Path Forward. JAMA 2019 Feb 26;321(8):743-4.

18. Ratwani RM, Hodgkins M, Bates DW. Improving Electronic Health Record Usability and Safety Requires Transparency. JAMA 2018 Dec 25;320(24):2533-4.

19. Sittig DF, Belmont E, Singh H. Improving the safety of health information technology requires shared responsibility: It is time we all step up. Healthcare 2018 Mar 1;6(1):7-12.

20. Tutty MA, Carlasare LE, Lloyd S, Sinsky CA. The complex case of EHRs: examining the factors impacting the EHR user experience. J Am Med Inform Assoc 2019 Jul 1;26(7):673-7.

21. Sinsky CA, Privitera MR. Creating a "Manageable 
Cockpit" for Clinicians: A Shared Responsibility. JAMA Intern Med 2018 Jun 1;178(6):741-2.

22. Gong Y, Kang H, Wu X, Hua L. Enhancing Patient Safety Event Reporting. A Systematic Review of System Design Features. Appl Clin Inform 2017 Aug 30;8(3):893-909.

23. Lyles CR, Fruchterman J, Youdelman M, Schillinger D. Legal, Practical, and Ethical Considerations for Making Online Patient Portals Accessible for All. Am J Public Health 2017;107(10):1608-11.

24. Powell KR. Patient-Perceived Facilitators of and Barriers to Electronic Portal Use: A Systematic Review. Comput Inform Nurs CIN 2017 Nov;35(11):565-73.

25. Antonio MG, Petrovskaya O, Lau F. Is research on patient portals attuned to health equity? A scoping review. J Am Med Inform Assoc [Internet]. [cited 2019 Jul 19] https://academic.oup.com/jamia/advance-article/doi/10.1093/jamia/ocz054/5487071

26. Walker J, Leveille S, Bell S, Chimowitz H, Dong Z, Elmore JG, et al. OpenNotes After 7 Years: Patient Experiences With Ongoing Access to Their Clinicians' Outpatient Visit Notes. J Med Internet Res 2019;21(5):e13876.

27. Hunter RF, Gough A, O'Kane N, McKeown G, Fitzpatrick A, Walker T, et al. Ethical Issues in Social Media Research for Public Health. Am J Public Health 2018 Jan 18;108(3):343-8.

28. Golder S, Ahmed S, Norman G, Booth A. Attitudes Toward the Ethics of Research Using Social Media: A Systematic Review. J Med Internet Res 2017;19(6):e195.

29. Gelinas L, Pierce R, Winkler S, Cohen IG, Lynch HF, Bierer BE. Using Social Media as a Research Recruitment Tool: Ethical Issues and Recommendations. Am J Bioeth 2017 Mar;17(3):3-14.

30. Crawford S, Hokke S, Nicholson J, Zion L, Lucke J, Keyzer P, et al. "It's not black and white": Public health researchers' and ethics committees' perceptions of engaging research participants online. Internet Res 2019 Jan 1;29(1):123-43.

31. Bender JL, Cyr AB, Arbuckle L, Ferris LE. Ethics and Privacy Implications of Using the Internet and Social Media to Recruit Participants for Health Research: A Privacy-by-Design Framework for Online Recruitment. J Med Internet Res 2017;19(4):e104.

32. Golder S, Scantlebury A, Christmas H. Understanding Public Attitudes Toward Researchers Using Social Media for Detecting and Monitoring Adverse Events Data: Multi Methods Study. J Med Internet Res 2019;21(8):e7081.

33. Chiauzzi E, Wicks P. Digital Trespass: Ethical and Terms-of-Use Violations by Researchers Accessing Data From an Online Patient Community. J Med Internet Res 2019;21(2):e11985.

34. Rieger A, Gaines A, Barnett I, Baldassano CF, Connolly Gibbons MB, Crits-Christoph P. Psychiatry Outpatients' Willingness to Share Social Media Posts and Smartphone Data for Research and Clinical Purposes: Survey Study. JMIR Form Res 2019 Aug 29;3(3):e14329.

35. Young SD, Garett R. Ethical Issues in Addressing Social Media Posts About Suicidal Intentions During an Online Study Among Youth: Case Study. JMIR Ment Health 2018;5(2):e33.

36. Terrasse M, Gorin M, Sisti D. Social Media,
E-Health, and Medical Ethics. Hastings Cent Rep 2019 Jan;49(1):24-33.

37. Barreto JE, Whitehair CL. Social Media and Web Presence for Patients and Professionals: Evolving Trends and Implications for Practice. PM R 2017 May;9(5S):S98-S105

38. Robledo I, Jankovic J. Media hype: Patient and scientific perspectives on misleading medical news. Mov Disord Off J Mov Disord Soc 2017 Sep;32(9):1319-23.

39. Rozenblum R, Greaves F, Bates DW. The role of social media around patient experience and engagement. BMJ Qual Saf 2017;26(10):845-8.

40. Ford E, Curlewis K, Wongkoblap A, Curcin V. Public Opinions on Using Social Media Content to Identify Users With Depression and Target Mental Health Care Advertising: Mixed Methods Survey. JMIR Ment Health 2019 Nov 13;6(11):e12942.

41. Zhou L, Bao J, Watzlaf V, Parmanto B. Barriers to and Facilitators of the Use of Mobile Health Apps From a Security Perspective: Mixed-Methods Study. JMIR MHealth UHealth 2019;7(4):e11223.

42. Zhou L, Parmanto B, Alfikri Z, Bao J. A Mobile App for Assisting Users to Make Informed Selections in Security Settings for Protecting Personal Health Data: Development and Feasibility Study. JMIR MHealth UHealth 2018;6(12):e11210

43. Hutton L, Price BA, Kelly R, McCormick C, Bandara AK, Hatzakis T, et al. Assessing the Privacy of mHealth Apps for Self-Tracking: Heuristic Evaluation Approach. JMIR MHealth UHealth 2018;6(10):e185.

44. Das G, Cheung C, Nebeker C, Bietz M, Bloss C. Privacy Policies for Apps Targeted Toward Youth: Descriptive Analysis of Readability. JMIR MHealth UHealth 2018;6(1):e3.

45. Powell A, Singh P, Torous J. The Complexity of Mental Health App Privacy Policies: A Potential Barrier to Privacy. JMIR MHealth UHealth 2018;6(7):e158.

46. Rendina HJ, Mustanski B. Privacy, Trust, and Data Sharing in Web-Based and Mobile Research: Participant Perspectives in a Large Nationwide Sample of Men Who Have Sex With Men in the United States. J Med Internet Res 2018;20(7):e233

47. Nebeker C, Murray K, Holub C, Haughton J, Arredondo EM. Acceptance of Mobile Health in Communities Underrepresented in Biomedical Research: Barriers and Ethical Considerations for Scientists. JMIR MHealth UHealth 2017;5(6):e87.

48. Cvrkel T. The ethics of mHealth: Moving forward. J Dent 2018;74 Suppl 1:S15-S20.

49. Di Matteo D, Fine A, Fotinos K, Rose J, Katzman M. Patient Willingness to Consent to Mobile Phone Data Collection for Mental Health Apps: Structured Questionnaire. JMIR Ment Health 2018;5(3):e56.

50. Shaw F. Mental health support apps and 'proper distance': relational ethics in mHealth. Media Int Aust 2019 May 1;171(1):9-22.

51. Martinez-Martin N, Kreitmair K. Ethical Issues for Direct-to-Consumer Digital Psychotherapy Apps: Addressing Accountability, Data Protection, and Consent. JMIR Ment Health 2018;5(2):e32.

52. Weizenbaum J. ELIZA - a Computer Program for the Study of Natural Language Communication Between Man and Machine. Commun ACM 1966 Jan;9(1):36-45.
53. Weizenbaum J. Computer Power and Human Reason: From Judgment to Calculation. New York, NY, USA: W. H. Freeman \& Co.; 1976.

54. Kretzschmar K, Tyroll H, Pavarini G, Manzini A, Singh I; NeurOx Young People's Advisory Group. Can Your Phone Be Your Therapist? Young People's Ethical Perspectives on the Use of Fully Automated Conversational Agents (Chatbots) in Mental Health Support. Biomed Inform Insights. 2019;11:1178222619829083.

55. Sage A, Blalock SJ, Carpenter D. Extending FDA guidance to include consumer medication information (CMI) delivery on mobile devices. Res Soc Adm Pharm 2017 Feb;13(1):209-13.

56. Niimi Y, Ota K. Examination of an Electronic Patient Record Display Method to Protect Patient Information Privacy. Comput Inform Nurs 2017 Feb;35(2):100-8.

57. Alaqra AS, Fischer-Hübner S, Framner E. Enhancing Privacy Controls for Patients via a Selective Authentic Electronic Health Record Exchange Service: Qualitative Study of Perspectives by Medical Professionals and Patients. J Med Internet Res 2018;20(12):e10954.

58. Finley EP, Schneegans S, Tami C, Pugh MJ, McGeary D, Penney L, Sharpe Potter J. Implementing prescription drug monitoring and other clinical decision support for opioid risk mitigation in a military health care setting: a qualitative feasibility study. J Am Med Inform Assoc 2018 May 1;25(5):515-22.

59. Theis RP, Malik AM, Thompson LA, Shenkman EA, Pbert L, Salloum RG. Considerations of Privacy and Confidentiality in Developing a Clinical Support Tool for Adolescent Tobacco Prevention: Qualitative Study. JMIR Form Res 2019;3(2):e12406

60. Dannenberg MD, Bienvenida JCM, Bruce ML, Nguyen T, Hinn M, Matthews J, et al. End-user views of an electronic encounter decision aid linked to routine depression screening. Patient Educ Couns 2019;102(3):555-63.

61. Epstein DA, Lee NB, Kang JH, Agapie E, Schroeder J, Pina LR, et al. Examining Menstrual Tracking to Inform the Design of Personal Informatics Tools. Proc 2017 CHI Conf Hum Factors Comput Syst [Internet]. New York, NY, USA: ACM; 2017 [cited 2019 Nov 13] http://doi.acm. org $/ 10.1145 / 3025453.3025635$. p. 6876-88.

62. Dexheimer JW, Greiner MV, Beal SJ, Johnson D, Kachelmeyer A, Vaughn LM. Sharing personal health record data elements in protective custody: youth and stakeholder perspectives. J Am Med Inform Assoc 2019 Aug 1;26(8-9):714-21.

63. Collins S, Dykes P, Bates DW, Couture B, Rozenblum R, Prey J, et al. An informatics research agenda to support patient and family empowerment and engagement in care and recovery during and after hospitalization. J Am Med Inform Assoc 2018 01;25(2):206-9.

64. King AC, Campero I, Sheats JL, Castro Sweet CM, Garcia D, Chazaro A, et al. Testing the comparative effects of physical activity advice by humans vs. computers in underserved populations: The COMPASS trial design, methods, and baseline characteristics. Contemp Clin Trials 2017;61:115-25.

65. Haimson OL. Mapping gender transition sentiment patterns via social media data: toward decreasing 
transgender mental health disparities. J Am Med Inform Assoc [Internet]. [cited 2019 Jul 19] https://academic.oup.com/jamia/advance-article/ doi/10.1093/jamia/ocz056/5497782; doi:10.1093/ jamia/ocz056

66. Harle CA, Golembiewski EH, Rahmanian KP, Krieger JL, Hagmajer D, Mainous AG, et al. Patient preferences toward an interactive e-consent application for research using electronic health records. J Am Med Inform Assoc 2018 Mar 1;25(3):360-8.

67. Abujarad F, Alfano S, Bright TJ, Kannoth S, Grant N, Gueble M, et al. Building an Informed Consent Tool Starting with the Patient: The Patient-Centered Virtual Multimedia Interactive Informed Consent (VIC). AMIA Annu Symp Proc 2017;2017:374-83

68. Harle CA, Golembiewski EH, Rahmanian KP, Brumback B, Krieger JL, Goodman KW, Mainous et al. Does an interactive trust-enhanced electronic consent improve patient experiences when asked to share their health records for research? A randomized trial. J Am Med Inform Assoc 2019 Jul 1;26(7):620-9.

69. Ramos SR. User-Centered Design, Experience, and Usability of an Electronic Consent User Interface to Facilitate Informed Decision-Making in an HIV Clinic. Comput Inform Nurs 2017 Nov;35(11):556-64.

70. Gilbert M, Bonnell A, Farrell J, Haag D, Bondyra $\mathrm{M}$, Unger D, et al. Click yes to consent: Acceptability of incorporating informed consent into an internet-based testing program for sexually transmitted and blood-borne infections. Int J Med Inform 2017;105:38-48.

71. Antal H, Bunnell HT, McCahan SM, Pennington C, Wysocki T, Blake KV. A cognitive approach for design of a multimedia informed consent video and website in pediatric research. J Biomed Inform 2017;66:248-58.

72. Moore S, Tassé A-M, Thorogood A, Winship I, Zawati M, Doerr M. Consent Processes for Mobile App Mediated Research: Systematic Review. JMIR MHealth UHealth 2017;5(8):e126.

73. Toscos T, Drouin M, Pater J, Flanagan M, Pfafman R, Mirro MJ. Selection biases in technology-based intervention research: patients' technology use relates to both demographic and health-related inequities. J Am Med Inform Assoc [Internet]. [cited $2019 \mathrm{Jul}$ 19] https://academic.oup.com/jamia/advance-article/doi/10.1093/jamia/ocz058/5512224

74. Kannan V, Wilkinson KE, Varghese M, LynchMedick S, Willett DL, Bosler TA, et al. Count me in: using a patient portal to minimize implicit bias in clinical research recruitment. J Am Med Inform Assoc [Internet] [cited 2019 Jul 19] https://academic.oup.com/jamia/advance-article/ doi/10.1093/jamia/ocz038/5488721

75. Feldmeth G, Naureckas ET, Solway J, Lindau ST. Embedding research recruitment in a community resource e-prescribing system: lessons from an implementation study on Chicago's South Side. JAm Med Inform Assoc 2019 Aug 1;26(8-9):840-6.

76. Lunn MR, Lubensky M, Hunt C, Flentje A, Capriotti MR, Sooksaman C, et al. A digital health research platform for community engagement, recruitment, and retention of sexual and gender minority adults in a national longitudinal cohort study - The PRIDE Study. J Am Med Inform Assoc [Internet] [cited 2019 Jul 19] https://academic. oup.com/jamia/advance-article/doi/10.1093/jamia/ ocz082/5509461

77. Reuter K, Zhu Y, Angyan P, Le N, Merchant AA,
Zimmer M. Public Concern About Monitoring Twitter Users and Their Conversations to Recruit for Clinical Trials: Survey Study. J Med Internet Res 2019;21(10):e15455.

78. Petersen C, Austin RR, Backonja U, Campos $\mathrm{H}$, Chung AE, Hekler EB, et al. Citizen science to further precision medicine: from vision to implementation. JAMIA Open [Internet]. [cited 2020 Apr 13] https://academic.oup.com/jamiaopen/advance-article/doi/10.1093/jamiaopen/ ooz060/5651081;

79. Stupp C. EU to Issue Guidance on Making Coronavirus Tracking Data Anonymous. Wall Str J [Internet]. 2020 Apr 10 [cited 2020 Apr 13] https://www.wsj.com/articles/eu-to-issue-guidance-on-making-coronavirus-tracking-data-anonymous-11586511004

80. Cahan E. Why telehealth can't significantly flatten the coronavirus curve - yet [Internet]. TechCrunch [cited 2020 Apr 13] https://social.techcrunch.com/2020/04/04/why-telehealth-cant-significantly-flatten-the-coronavirus-curve-yet/

Correspondence to:

Harry Hochheiser, PhD

University of Pittsburgh School of Medicine

5607 Baum Boulevard

Pittsburgh, PA 15206-3701

USA

Tel: +14126489300

Fax:+14126245310

E-mail: harryh@pitt.edu 